\title{
Defect-mediated transport and electronic irradiation effect in individual domains of CVD-grown monolayer $\mathrm{MoS}_{2}$
}

\author{
Corentin Durand, Xiaoguang Zhang, and Jason Fowlkes \\ Center for Nanophase Materials Sciences, Oak Ridge National Laboratory, Oak Ridge, Tennessee 37831 \\ Sina Najmaei and Jun Lou \\ Department of Materials Science and NanoEngineering, Rice University, Houston, Texas 77251 \\ An-Ping Li ${ }^{\text {a) }}$ \\ Center for Nanophase Materials Sciences, Oak Ridge National Laboratory, Oak Ridge, Tennessee 37831
}

(Received 10 October 2014; accepted 7 January 2015; published 16 January 2015)

\begin{abstract}
The authors study the electrical transport properties of atomically thin individual crystalline grains of $\mathrm{MoS}_{2}$ with four-probe scanning tunneling microscopy. The monolayer $\mathrm{MoS}_{2}$ domains are synthesized by chemical vapor deposition on $\mathrm{SiO}_{2} / \mathrm{Si}$ substrate. Temperature dependent measurements on conductance and mobility show that transport is dominated by an electron charge trapping and thermal release process with very low carrier density and mobility. The effects of electronic irradiation are examined by exposing the film to electron beam in the scanning electron microscope in an ultrahigh vacuum environment. The irradiation process is found to significantly affect the mobility and the carrier density of the material, with the conductance showing a peculiar time-dependent relaxation behavior. It is suggested that the presence of defects in active $\mathrm{MoS}_{2}$ layer and dielectric layer create charge trapping sites, and a multiple trapping and thermal release process dictates the transport and mobility characteristics. The electron beam irradiation promotes the formation of defects and impact the electrical properties of $\mathrm{MoS}_{2}$. Our study reveals the important roles of defects and the electron beam irradiation effects in the electronic properties of atomic layers of $\mathrm{MoS}_{2}$. C 2015 American Vacuum Society. [http://dx.doi.org/10.1116/1.4906331]
\end{abstract}

\section{INTRODUCTION}

Transition metal dichalcogenides such as molybdenum disulfide $\left(\mathrm{MoS}_{2}\right)$ have attracted great interest as candidates to fill the need of two dimensional semiconductor materials. By controlling the thickness, the bandgap of $\mathrm{MoS}_{2}$ thin films can be tuned from $1.2 \mathrm{eV}$ (bulk material, indirect bandgap) to $1.8 \mathrm{eV}$ (monolayer film, direct bandgap). ${ }^{1}$ Recently, large scale $\mathrm{MoS}_{2}$ monolayers have been grown by chemical vapor deposition (CVD) on silicon dioxide $\left(\mathrm{SiO}_{2}\right)$ substrate, ${ }^{2-4}$ showing the possibility of low cost scalable device fabrication. However, measurements on CVD grown $\mathrm{MoS}_{2}$ monolayers $^{2-4}$ reveal mobility values that are usually orders of magnitude lower than those (exceeding $200 \mathrm{~cm}^{2} \mathrm{~V}^{-1} \mathrm{~s}^{-1}$ ) reported on exfoliated $\mathrm{MoS}_{2}$ monolayers. ${ }^{5}$ The physical origin of the difference between CVD and exfoliated $\mathrm{MoS}_{2}$ is not clear at present; however, structural defects ${ }^{6}$ such as vacancies, dislocations, ${ }^{7}$ grain boundaries, ${ }^{4}$ as well as charged interfacial states due to the dielectrics in contact $^{8}$ can be responsible for the degradation in mobility. Although this problem has presented a major hurdle to the realization of wafer-scale $\mathrm{MoS}_{2}$ electronics and photonics, systematic studies of defects' role in individual crystal domains are still lacking.

Furthermore, it is known that the use of high-energy particle irradiation on semiconductor nanostructures can drastically modify their electronic properties. Durand et al. ${ }^{9}$ have shown that InAs nanowires exhibiting n-type semiconducting behavior turn metallic under electronic irradiation. On

${ }^{\text {a)} E l e c t r o n i c ~ m a i l: ~ a p l i @ o r n l . g o v ~}$
$\mathrm{MoS}_{2}$, long range magnetic ordering in bulk crystal ${ }^{10}$ has been demonstrated after irradiation with a $2 \mathrm{MeV}$ proton beam. At higher beam energy $(10 \mathrm{MeV})$, a time dependent transport behavior has been observed in $\mathrm{MoS}_{2}$ field effect transistors ${ }^{11}$ and related to the formation of charge traps in dielectric material and its interface with the $\mathrm{MoS}_{2}$ (few layers) channel. In the case of electronic irradiation, Komsa et $a .^{12}$ have reported that an electron beam energy about $90 \mathrm{keV}$ is necessary to create sulfur vacancies in $\mathrm{MoS}_{2}$ by knock-on mechanism. Such defect engineering presents a lot of attractive possibilities to tune the properties of this material and also to create new kinds of nanostructure, like $\mathrm{Mo}_{5} \mathrm{~S}_{4}$ nanoribbons. ${ }^{13,14}$ Recently, Zhou et al. ${ }^{6}$ suggest that vacancies can form even in the energy range provided by scanning electron microscope (SEM, $30 \mathrm{keV}$ ) under electron beam exposure. Although electron beam techniques are widely used in microscopic imaging and nanofabrication processes, the effect of electron beam irradiation on the transport properties of $\mathrm{MoS}_{2}$ atomic layer has not been evaluated, especially at low beam energy regime.

Here, we study the transport properties of CVD-grown monolayer on $\mathrm{SiO}_{2} / \mathrm{Si}$ substrate by directly measuring the resistivity and the mobility of the material with a field-effect transistor configuration with the use of a cryogenic fourprobe scanning tunneling microscope (STM). ${ }^{15,16}$ The combination of the STM scanners and a SEM enables us to connect the STM tips on individual $\mathrm{MoS}_{2}$ domains. The temperature dependent resistivity and mobility show a defect dominated transport process. Furthermore, the effects of electronic irradiation are examined by exposing the film to 
electron beam in the SEM in an ultrahigh vacuum environment, and it is found that a clear change of transport behavior of the material after electronic irradiation with SEM at low energy $(5 \mathrm{keV})$. It is suggested that the presence of defects create charge trapping states and leads to the low mobility, consistent with recent theoretical calculations ${ }^{17,18}$ where vacancies and antisites defects are shown to create localized gap states.

\section{EXPERIMENT}

The synthesis of monolayer $\mathrm{MoS}_{2}$ domains by CVD process has been described by Najmaei et al. ${ }^{2}$ Briefly, $\mathrm{MoO}_{3}$ nanoribbons and sublimated sulfur are used as precursors. The $\mathrm{MoO}_{3}$ nanoribbons are dispersed on silicon substrates and placed close to a bare $\mathrm{Si} / \mathrm{SiO}_{2}$ substrate designated for the growth of $\mathrm{MoS}_{2}$ at the center of a quartz tube (furnace) under a constant $\mathrm{N}_{2}$ flow of $200 \mathrm{sccm}$. And $0.8-1.2 \mathrm{~g}$ of sublimated sulfur is placed in a container at the opening of the furnace where the maximum temperature approximately reaches $600^{\circ} \mathrm{C}$. At a rate of approximately $20^{\circ} \mathrm{C} / \mathrm{min}$, the center of the furnace is gradually heated from room temperature to $550^{\circ} \mathrm{C}$ in $30 \mathrm{~min}$. The sulfur slowly evaporates when the temperature reaches approximately $550^{\circ} \mathrm{C}$. Then, at the slower ramping rate of $\sim 5^{\circ} \mathrm{C} / \mathrm{min}$, the chamber is heated to $850^{\circ} \mathrm{C}$. This temperature is maintained for $10-15 \mathrm{~min}$ and then the system is naturally cooled back to room temperature.

The as-grown sample is studied with a four-probe scanning tunneling microscope (4P-STM), a characterization platform combining four STM and a SEM operating in ultrahigh vacuum (UHV) ${ }^{15}$ (base pressure $<5 \times 10^{-10}$ Torr). The 4P-STM enables the positioning of four STM electrochemically etched tungsten tips in an arbitrary arrangement under SEM view. This tool provides the capability of establishing contacts on surfaces and nanostructures with the resolution offered by the STM scanners. ${ }^{19}$

STM tips can act as electrodes and provide good contacts on metals and some doped semiconductors. ${ }^{16}$ Here, our study focuses on single $\mathrm{MoS}_{2}$ monolayers lying on a $300 \mathrm{~nm}$ thick $\mathrm{SiO}_{2}$ substrate, a subnanometer thick semiconductor with a bandgap of around $1.8 \mathrm{eV}$ on an insulator. Unlike measurements on graphene, ${ }^{20}$ it turns out very challenging to establish reliable electrical contacts between the STM tips and the $\mathrm{MoS}_{2}$ by gentle contacts without damaging the monolayer. In order to ensure reliable electrical contacts, platinum pads have been deposited by using electron beam induced deposition (EBID) $)^{21}$ at several locations on $\mathrm{MoS}_{2}$ domains [Fig. 1(a)]. As reported by Das et al., ${ }^{22} \mathrm{Sc}$ and Ti would be better choices than Pt due to their lower work function values, which can facilitate the carrier injection process to the $\mathrm{MoS}_{2}$. Here, we choose Pt because of the availability of the sources in our EBID technique. However, as will be shown later, we still obtain an ohmic contact and the contact resistance has been taken into account in our analysis.

The EBID experiments were conducted in an FEI Nova 600 dual ion/electron microscope. A focused beam of electrons $(5 \mathrm{keV}, 1.6 \mathrm{nA}$, and diameter $\sim 300 \mathrm{~nm})$ was used to

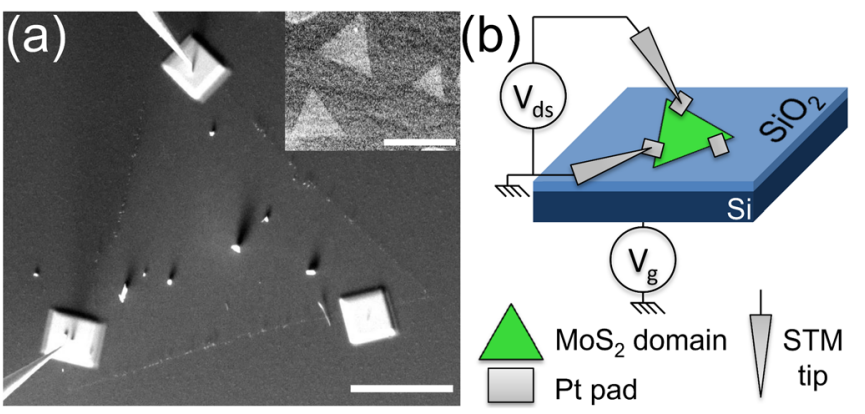

FIG. 1. (Color online) (a) SEM image of single $\mathrm{MoS}_{2}$ grain grown on a $\mathrm{SiO}_{2}$ substrate with three Pt pads deposited. Two STM tips are connected on the upper and left pads. Scale bar $10 \mu \mathrm{m}$. Inset: SEM image of single grains without Pt pad. Scale bar: $20 \mu \mathrm{m}$. (b) Schematics view of the measurement setup.

partially dissociate substrate absorbed, organometallic precursor molecules, resulting in a condensed deposit. Notably, secondary electrons, created during inelastic primary electron-substrate interactions, ultimately lead to precursor dissociation at the sample surface during EBID. The precursor molecule was a platinum based, organometallic compound trimethyl(methylcyclopentadienyl)platinum(IV) $\left[\mathrm{C}_{5} \mathrm{H}_{4} \mathrm{CH}_{3} \mathrm{Pt}\left(\mathrm{CH}_{3}\right)_{3}\right]$. The precursor was delivered to the $\mathrm{MoS}_{2}$ covered surface by an injection nozzle located $100 \mu \mathrm{m}$ above the sample surface. The nozzle makes it possible to achieve a relatively high local pressure of precursor ( $\sim 10$ mTorr) without compromising the high vacuum requirement in the electron optics column. The injection nozzle was $500 \mu \mathrm{m}$ diameter. The resulting deposit contained a mixture of Pt nanoparticles embedded in an amorphous carbon matrix. The composition of the deposit following EBID was $\sim \mathrm{PtC}_{5}$ according to qualitative energy dispersive spectroscopy measurements. Deposit composition was not quantified because the sample heating cycle, following EBID and required for sample preparation prior to conducting electrical measurements, was certain to remove additional carbon from the deposit, which is a welldocumented phenomenon that occurs when heating EBID deposits. ${ }^{21,23}$ Direct write experiments were initiated when the sample chamber was evacuated to a base pressure on the order of $\sim 7.5 \times 10^{-6}$ Torr. A deposit with typical lateral dimensions of $\sim 4 \mu \mathrm{m} \times 4 \mu \mathrm{m}$ was deposited by multiple raster scans of the electron beam. Care was taken to minimize electron exposure outside the deposition zone by avoiding electron beam imaging following deposition; only after flushing the chamber with $\mathrm{N}_{2}$, up to atmospheric pressure, was electron imaging conducted for characterization purposes. The deposition zone direct-write pattern was a square array of pixels with a pixel spacing of $73.3 \mathrm{~nm}$. The electron beam dwell time per pixel was $100 \mu$ s. Multiple beam passes (200) are used in order to avoid precursor surface depletion in the growth zone that can occur for relatively long beam dwell times or high beam current densities. This looping tactic effectively minimizes the dose of electrons, thereby minimizing $\mathrm{MoS}_{2}$ electron exposure. A final deposit height of $\sim 250 \mathrm{~nm}$ resulted. 
The sample is annealed at $135^{\circ} \mathrm{C}$ for $8 \mathrm{~h}$ in the load-lock of the UHV system (base pressure lower than $5 \times 10^{-8}$ Torr) before being transferred onto the STM platform. Recent studies $^{24,25}$ suggested a higher annealing temperature (above $250{ }^{\circ} \mathrm{C}$ ) of $\mathrm{MoS}_{2}$. Here, we anneal the sample at lower temperature for a longer time in order to minimize the effect of metal diffusion, a process not being well examined on monolayer CVD MoS ${ }_{2}$. The SEM image in the inset of Fig. 1(a) shows $\mathrm{MoS}_{2}$ monolayer islands grown on a $\mathrm{SiO}_{2}$ layer. Those domains have a triangular shape with a size ranging from a few micrometers to few tens of micrometers. The main SEM image shows a single domain on which Pt pads have been deposited at each corner of the triangle (bright squares). The STM probes are directly connected onto the Pt pads.

\section{RESULTS AND DISCUSSION}

The transport measurements are realized with the configuration depicted in Fig. 1(b). The source-drain current $\mathrm{I}_{\mathrm{ds}}$ versus the voltage $V_{\mathrm{ds}}$ is measured by the two probes in contact with the $\mathrm{MoS}_{2}$ domain whereas the back gate voltage $V_{\mathrm{g}}$ is applied to the heavily doped p-type $\mathrm{Si}$ substrate, with the thermally grown $\mathrm{SiO}_{2}$ serving as gate dielectric material.

At room temperature, the $I_{\mathrm{ds}}\left(V_{\mathrm{ds}}\right)$ curves is linear (Fig. 2) up to $20 \mathrm{~V}$ (not shown here) even with a gate voltage $V_{\mathrm{g}}$ ranging from -200 to $+200 \mathrm{~V}$, indicating that the Pt pads provide Ohmic contacts to the $\mathrm{MoS}_{2}$. The $I_{\mathrm{ds}}\left(V_{\mathrm{g}}\right)$ curve (Fig. 2) has typical characteristics for a n-type field effect transistor, as previously observed for $\mathrm{MoS}_{2}$ films grown with this method. ${ }^{2}$ In the linear region of the transfer characteristic $I_{\mathrm{ds}}\left(V_{\mathrm{g}}\right)$, the source-drain current $I_{\mathrm{ds}}$ is given by ${ }^{26,27}$

$$
I_{\mathrm{ds}} \cong \frac{W}{L} \times C \times \mu_{\mathrm{FE}} \times\left[\left(V_{\mathrm{g}}-V_{\mathrm{T}}\right) V_{\mathrm{ds}}-\frac{V_{\mathrm{ds}}^{2}}{2}\right],
$$

where $V_{\mathrm{T}}$ is a threshold voltage that can be deduced from the linear extrapolation of the $I_{\mathrm{ds}}\left(V_{\mathrm{g}}\right)$ characteristic, and we assume that the mobility is only slowly varying with $V_{\mathrm{g}}$ in

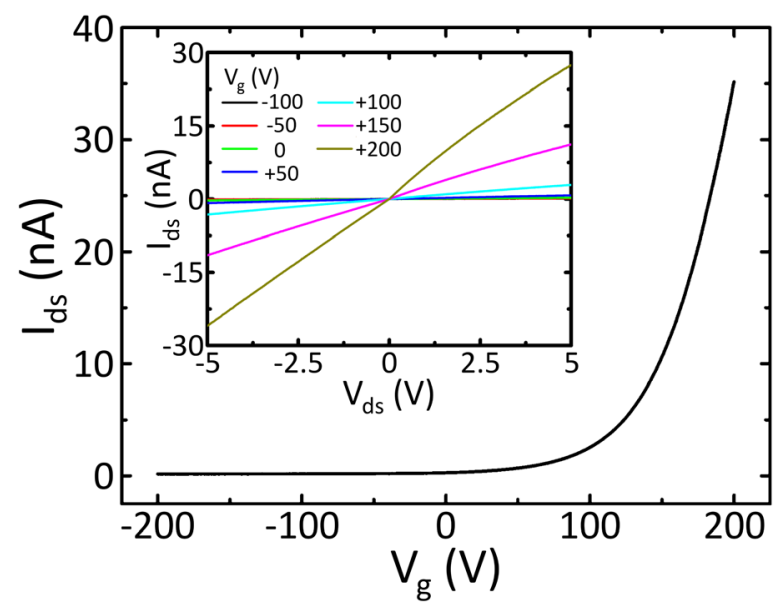

FIG. 2. (Color online) Room-temperature $I_{\mathrm{ds}}\left(V_{\mathrm{g}}\right)$ transfer characteristic for a single $\mathrm{MoS}_{2}$ domain with the field-effect transistor configuration described in the main text with $5 \mathrm{~V}$ applied bias voltage $V_{\mathrm{ds}}$. Inset: $I_{\mathrm{ds}}\left(V_{\mathrm{ds}}\right)$ characteristics at different gate voltage $V_{\mathrm{g}}$. the linear region of the $I_{\mathrm{ds}}\left(V_{\mathrm{g}}\right)$ characteristics. The square term of $V_{\mathrm{ds}}$ can be neglected under the condition $V_{\mathrm{g}} \gg V_{\mathrm{ds}}$. The field effect mobility $\left(\mu_{\mathrm{FE}}\right)$ is extracted from the transconductance $g_{\mathrm{m}}=d I_{\mathrm{ds}} / d V_{\mathrm{g}}$ using $\mathrm{s}^{5,26-28}$

$$
\mu_{\mathrm{FE}}=\frac{L}{C W} \times \frac{1}{V_{\mathrm{ds}}} \times g_{\mathrm{m}},
$$

where $L$ and $W$ are the length and the width of the channel, respectively, and $C$ is the normalized gate capacitance per unit area. The measured mobility is $0.029 \mathrm{~cm}^{2} \mathrm{~V}^{-1} \mathrm{~s}^{-1}$, a low value compared to previous reports on large $\mathrm{MoS}_{2}$ films. ${ }^{2,3}$ The temperature dependent transport behaviors are measured from 140 to $315 \mathrm{~K}$. Figure 3(a) shows the evolution of the mobility as a function of the temperature at $V_{\mathrm{g}}=200 \mathrm{~V}$. The measured $\mu_{\mathrm{FE}}$ (solid squares) increases with the temperature, as expected for a semiconductor.

The mobility can also be calculated from the channel conductance using ${ }^{26}$

$$
g_{d}=\frac{d I_{\mathrm{ds}}}{d V_{\mathrm{ds}}}=\frac{W}{L} \times \mu_{\mathrm{FE}} \times C \times\left(V_{\mathrm{g}}-V_{\mathrm{T}}\right) .
$$

However, the two terminal configurations we use implies that our measurements are affected by the contact resistances $R_{C}$ as compared to mobility measurements with a multiterminal configuration. ${ }^{29,30}$ Following Horowitz et al., ${ }^{27}$ we introduce the contact resistances $R_{C}$ in the expression of the source-drain current $I_{\mathrm{ds}}$ (the square term of $V_{\mathrm{ds}}$ being neglected) by changing $V_{\mathrm{ds}}$ to $V_{\mathrm{ds}}-I_{\mathrm{ds}} \times R_{C}$. Then, the expression for the channel conductance Eq. (3) becomes

$$
g_{\mathrm{d}}=\frac{(W / L) C \mu_{\mathrm{eff}}\left(V_{\mathrm{g}}-V_{\mathrm{T}}\right)}{1+(W / L) C \mu_{\mathrm{eff}} R_{C}\left(V_{g}-V_{\mathrm{T}}\right)},
$$

$\mu_{\text {eff }}$ being the "effective mobility." As suggested by Horowitz et al. ${ }^{27}$ the mobility can be assumed to follow an empirical law of the form

$$
\mu_{\text {eff }}=\alpha\left(V_{\mathrm{g}}-V_{\mathrm{T}}\right)^{\beta} .
$$

Performing a least squares fit of the data gives us an estimation of the contact resistances $R_{C}$. The contact resistances (not shown) is found to decrease with the increasing temperature and account for $10 \%$ to $40 \%$ of the total resistance in our temperature range and $V_{\mathrm{g}}$ from 100 to $200 \mathrm{~V}$. Once we have estimated the contact resistances, we recalculate the effective mobility $\mu_{\text {eff }}$ from the channel conductance using Eq. (4). The values at $V_{\mathrm{g}}=200 \mathrm{~V}$ are shown in Fig. 3(a), represented by the empty squares. One can see that $\mu_{\text {eff }}$ is higher by a factor 2-3 than $\mu_{\mathrm{FE}}$, which means that the contact resistances do play a role in our system. Moreover, the mobility changes slightly with the applied gate bias after considering the contact resistance even for $V_{\mathrm{g}} \gg V_{\mathrm{ds}}$ as shown in Fig. 3(b).

Above analysis indicates that the mobility in $\mathrm{MoS}_{2}$ has a strong temperature dependence as well as a weak gate voltage dependence. This is consistent with a multiple trapping and release (MTR) model described by Horowitz et $\mathrm{al}^{27}$ The MTR model requires that most of the charge carriers are 

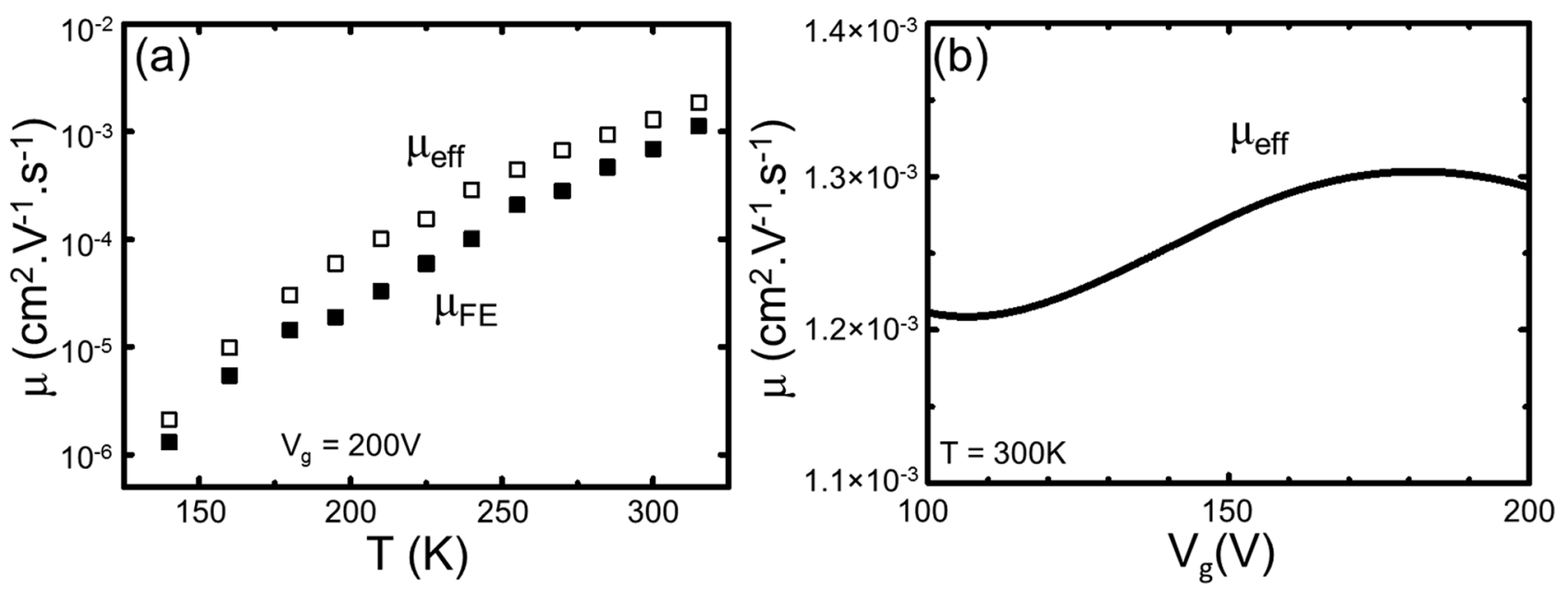

FIG. 3. (a) Field-effect $\mu_{\mathrm{FE}}$ mobility (solid squares) as a function of temperature extracted at $V_{\mathrm{g}}=200 \mathrm{~V}$ from the transconductance $g_{\mathrm{m}}$ as a function of the temperature [Eq. (2)]. The empty squares represent the effective mobility $\mu_{\mathrm{eff}}$ (same $V_{\mathrm{g}}$ ) calculated with the channel conductance Eqs. (4) and (5). (b) The effective mobility as a function of gate bias voltage at $300 \mathrm{~K}$ after considering the contact resistances [Eqs. (4) and (5)].

trapped in localized states. The amount of released charge carriers to an extended-state that contributes to transport depends on the energy level of the localized states, the temperature, and the gate voltage, leading to a thermally activated mobility. In such a trap-limited transport model, transport occurs only in delocalized bands. This is in sharp contrast to the mechanism of variable range hopping, which is often invoked as the transport mechanism in $\mathrm{MoS}_{2}$, where all trap levels participate in hopping conduction and contribution from delocalized carriers is absent.

Figure 4 shows the effective mobility $\mu_{\text {eff }}$ at several different $V_{\mathrm{g}}$ as a function of the inverse of the temperature, $R_{\mathrm{C}}$ being subtracted. Indeed, $\mu_{\text {eff }}$ can be modeled with thermally activated transport:

$$
\mu_{\text {eff }} \sim \exp \left[-\frac{E_{\mathrm{a}}}{k_{\mathrm{B}} T}\right],
$$

where $E_{\mathrm{a}}$ is the activation energy and $k_{\mathrm{B}}$ the Boltzmann constant. With $V_{\mathrm{g}}$ increasing, the activation energy $E_{\mathrm{a}}$ decreases slightly (from 150 for $144 \mathrm{meV}$ when $V_{\mathrm{g}}$ increases from 140

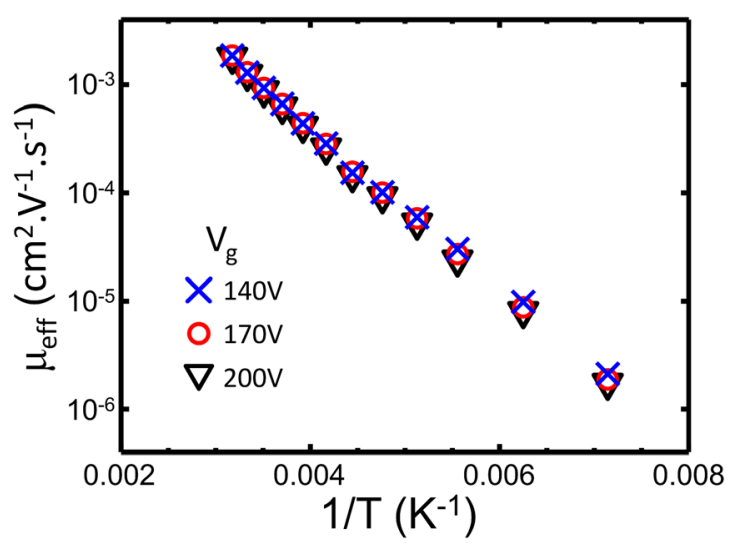

FIG. 4. (Color online) Effective mobility as a function of the inverse of the temperature at different gate voltage. Arrhenius plots give activation energy in the range of $144-150 \mathrm{meV}$. to $200 \mathrm{~V}$ ), in agreement with the MTR model and previously reported behaviors of $\mathrm{MoS}_{2} \cdot{ }^{17,29,30}$ Note, we have tried to fit the temperature dependence of $\mu$ and of the resistivity $\rho$ with the variable-range hopping equation for $2 \mathrm{D}$ materials

$$
\mu_{\text {eff }} \sim \exp \left[-\left(\frac{T_{0}}{T}\right)^{\frac{1}{3}}\right]
$$

and

$$
\rho \sim \exp \left[\left(\frac{T_{0}}{T}\right)^{\frac{1}{3}}\right]
$$

and obtained a fitting parameter $T_{0}=2.8 \times 10^{6} \mathrm{~K}$ that is orders of magnitude higher than observed for exfoliated $\mathrm{MoS}_{2}{ }^{8,}$,17,31

The mobility values presented above are very low compared to the intrinsic limit reported for $\mathrm{MoS}_{2}$ monolayers $\left(410 \mathrm{~cm}^{2} \mathrm{~V}^{-1} \mathrm{~s}^{-1}\right){ }^{32}$ TEM studies on CVD grown $\mathrm{MoS}_{2}$ islands similar to what we used in this work ${ }^{2}$ showed the good quality of the monolayer islands, and also revealed the presence of different structural defects like sulfur vacancies. ${ }^{6}$ In addition to the trap states at the interface between the dielectric and the channel, ${ }^{8}$ these structural defect can lead to localized electronic states. ${ }^{6,17}$ In their recent studies, Zhu et al. ${ }^{30}$ demonstrated that the presence of a large amount of localized traps leads to the smearing of the band edge and the formation of band tail states in the $\mathrm{MoS}_{2}$ energy gap. The mobility edge forms a boundary in the band tail, which separates extended states with band transport and localized states that conduct via thermally assisted mechanisms. Based on the subthreshold swing $(S S)$ extracted from $I_{\mathrm{ds}}\left(V_{\mathrm{g}}\right)$ curves, we can estimate the value of trap density $D_{\text {it }}$ with the formula ${ }^{26}$

$$
\begin{aligned}
S S & \cong \ln 10 \times \frac{k T}{q}\left(1+\frac{C_{\mathrm{D}}+C_{\mathrm{it}}}{C}\right) \\
& =\ln 10 \times \frac{k T}{q}\left(1+\frac{C_{\mathrm{D}}+q D_{\mathrm{it}}}{C}\right),
\end{aligned}
$$


where $C_{\mathrm{D}}$ and $C_{\mathrm{it}}$ are the capacitance associated to the depletion-layer and the traps, respectively, and $q$ the elementary charge. For this device, $S S$ is very large, in the range of 225-275 V/dec, compared to other reports on CVD $\mathrm{MoS}_{2}$ monolayers ( $200 \mathrm{mV} / \mathrm{dec}$ for Ref. 30, $13 \mathrm{~V} / \mathrm{dec}$ in Ref. 3). In that case, we assume $C_{\mathrm{it}} \gg C_{\mathrm{D}}$ and find a density of trap state in the $10^{14} \mathrm{eV}^{-1} \mathrm{~cm}^{-2}$ range. Such a high value of $D_{\mathrm{it}}$ corroborates the MTR model.

To further examine the role of defects in the transport of $\mathrm{MoS}_{2}$, we now study the impact of the electronic irradiation. Figure 5(a) shows the conductance measured at room temperature and $V_{\mathrm{g}}=100 \mathrm{~V}$ as a function of time with several irradiation events with a $5 \mathrm{kV}$ electron beam. Since the connection of the STM tips onto the Pt requires real time SEM imaging, the first measurement (black star and black curve in the inset) has been performed around $30 \mathrm{~min}$ after electron beam irradiation (first irradiation). During transport measurements, the electron beam is turned off. After one additional SEM imaging scan $(\sim 2$ min for the whole frame, second irradiation event indicated in red arrow), the conductance is multiplied by a factor 2, as shown by the first red square in Fig. 5(a) and
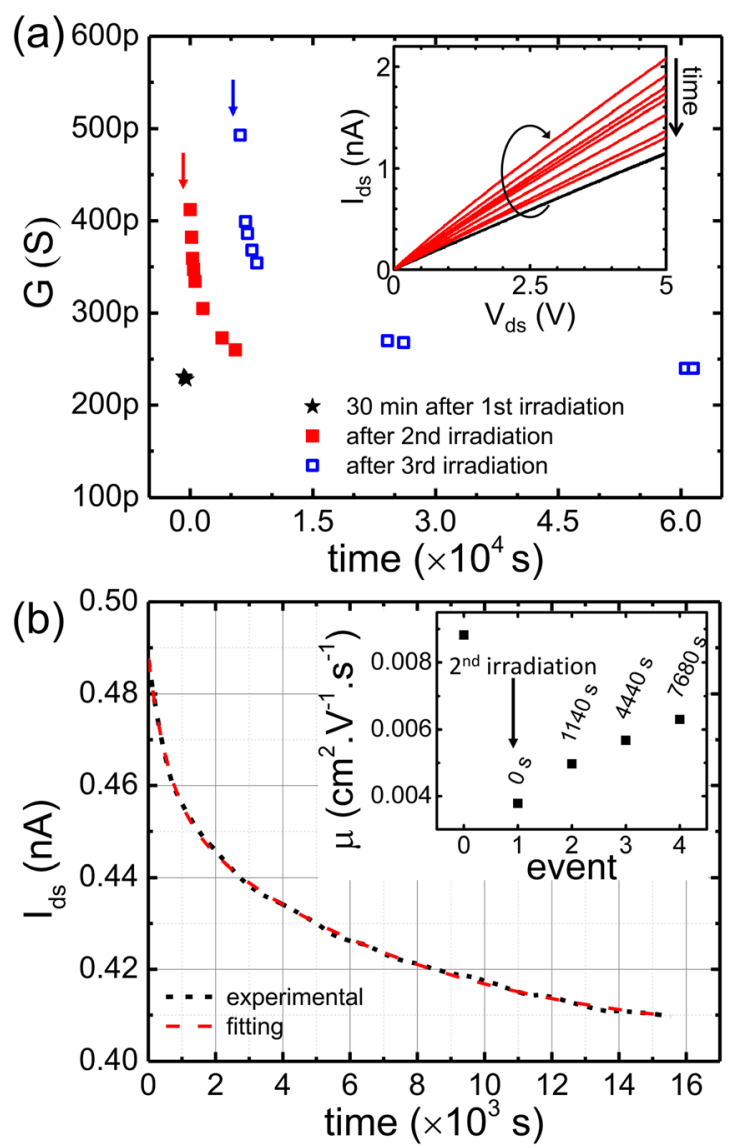

FIG. 5. (Color online) (a) Time dependence of the conductance measured at $V_{\mathrm{g}}=+100 \mathrm{~V}$ after three irradiation events. Inset: $I_{\mathrm{ds}}\left(V_{\mathrm{ds}}\right)$ curves after first and second irradiation events, corresponding to the black star and the solid squares of the main graph, respectively. (b) $I_{\mathrm{ds}}$ vs time measured at $V_{\mathrm{ds}}=5 \mathrm{~V}$ and $V_{\mathrm{g}}=0 \mathrm{~V}$ after irradiation $\left[\mathrm{MoS}_{2}\right.$ domain different from (a)]. The dashed curve is fitted with $y=y_{0}+A_{1} e^{-\left(x-x_{0}\right) / t_{1}}+A_{2} e^{-\left(x-x_{0}\right) / t_{2}}$, where the times constants $t_{1}=600 \mathrm{~s}$ and $t_{2}=7100 \mathrm{~s}$. Inset: Mobility values of the same domain before and after second irradiation event (arrow) measured at different time as indicated. upper red $\mathrm{I}_{\mathrm{ds}}\left(V_{\mathrm{ds}}\right)$ curves in the inset. The conductance becomes time dependent with a slow relaxation process. Another factor 2 of increase of conductance is observed after the third irradiation event [Fig. 5(a), upper arrow], which then starts to relax and reaches its original value after $6 \times 10^{4} \mathrm{~s}$ [Fig. 5(a), blue squares]. Figure 5(b) shows the time dependence of the current on another $\mathrm{MoS}_{2}$ domain at $V_{\mathrm{ds}}=5 \mathrm{~V}$, $V_{\mathrm{g}}=0 \mathrm{~V}$ and room temperature. The measurement starts several seconds after an irradiation event and shows that the current decreases by about $20 \%$ after $15 \times 10^{3} \mathrm{~s}$. The mobility [Fig. 5(b), inset] drops noticeably after the second irradiation event (marked by the black arrow) and then gradually recovers its initial value. Thus, the rise in the conductivity after irradiation [Fig. 5(a)] is due to an increase of the carrier concentration. Unlike the InAs nanowires which turn metallic, ${ }^{9}$ monolayer $\mathrm{MoS}_{2}$ keeps semiconducting. Moreover, the irradiation effect has not been observed at $T \leq 140 \mathrm{~K}$ for $\mathrm{MoS}_{2}$, and the current recovers its original value instantaneously without decaying process.

The subthreshold swing of the device that is irradiated with an electron beam becomes worse, as shown in Fig. 6(a). Such degradation of the subthreshold swing after irradiation is similar to that observed on $\mathrm{MoS}_{2}$ irradiated by proton beams ${ }^{11}$ and can be attributed to the irradiation-induced trap states at the interface of $\mathrm{MoS}_{2}$ and $\mathrm{SiO}_{2}$. Based on the subthreshold swing extracted from $I_{\mathrm{ds}}\left(V_{\mathrm{g}}\right)$ curves, we can estimate the value of trap density $D_{\mathrm{it}}$ after the irradiation event as plotted in Fig. 6(b) (solid squares), which are even higher than observed before irradiation. Moreover, as shown in Fig. 6(b) (empty squares, right axis), the threshold voltage $V_{\text {th }}$ shifts toward negative value after irradiation (event 1 in red), indicating an increase of the carrier concentration, in agreement with the observation on conductance change.

The interface trap states induced by irradiation process affect the Fermi level position of the conduction channel and thus the carrier concentration, which is consistent with our observed shift of the threshold voltage and those reported by Kim et al. ${ }^{11}$ Moreover, we find that the interface trap states and the carrier density vary over a different time scale. Figure 6(b) shows that the interface trap density increase from $1.42 \times 10^{14} \mathrm{~cm}^{-2} \mathrm{eV}^{-1}$ (event 0 , not shown on the graph) to $1.67 \times 10^{14} \mathrm{~cm}^{-2} \mathrm{eV}^{-1}$ after irradiation (event 1 , red filled square) and then, following exponential decay with a time constant $\sim 600 \mathrm{~s}$. On the other side, the carrier concentration, related to the threshold voltage shift $\Delta V_{\text {th }}$ [Fig. 6(b), empty squares], varies with a much higher the time constant of $\sim 2600 \mathrm{~s}$. Thus, the interface trap states density alone cannot account for the change of carrier concentration in the conduction channel. Interestingly, the conductance change after irradiation also shows a two component decay behavior [as shown with the fitting curve in Fig. 5(b)] and one component has almost the same time constant as the interface trap density. All these observations show that both the defects created in active $\mathrm{MoS}_{2}$ layer and trap states at the interface of $\mathrm{MoS}_{2} / \mathrm{SiO}_{2}$ are responsible for the peculiar conductance relaxation process. We would like to mention that we have studied the influence of the electron beam energy on the $\mathrm{I}(\mathrm{V})$ characteristics of $\mathrm{MoS}_{2}$ by varying the 

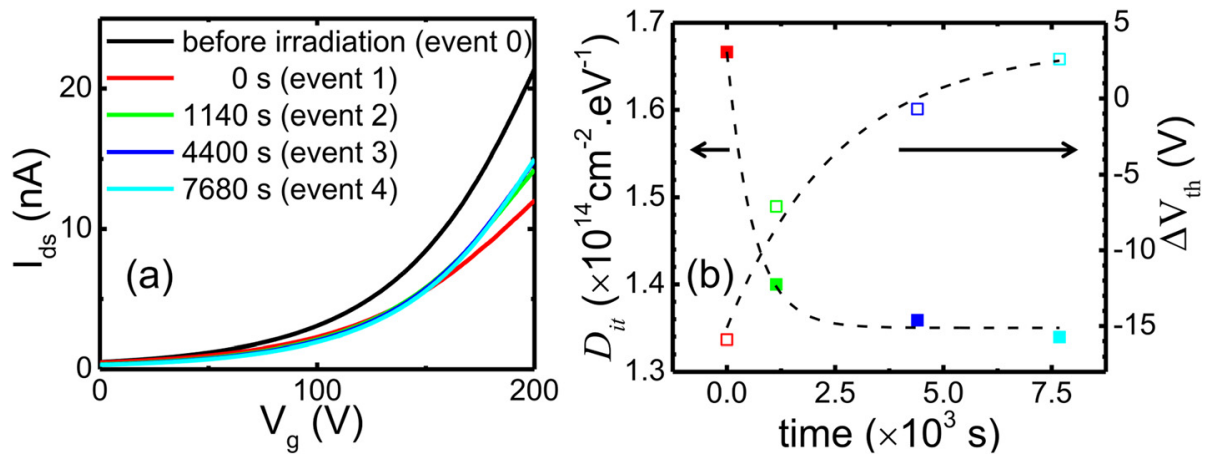

FIG. 6. (Color online) (a) $I_{\mathrm{ds}}\left(V_{\mathrm{g}}\right)$ characteristics before (upmost curve) and after (lower curves) an irradiation event (same dataset as the inset of Fig. 4(b) with event number reported). The lowest curve (event 1$)$ is measured right after the irradiation event $(0 \mathrm{~s})$. (b) Trap density $D_{\text {it }}$ (filled squares, left axis) deduced from the subthreshold swing and shift of the threshold voltage $\Delta V_{\text {th }}$ (empty squares, right axis) after irradiation extracted from (a) with corresponding colors (event 0 not shown). The dashed lines are fitting curves with $y=y_{1}+A_{3} e^{-\left(x-x_{1}\right) / t_{3}}$ and time constant $t_{3}=600$ and $2600 \mathrm{~s}$ for $D_{\mathrm{it}}$ and $\Delta V_{\mathrm{th}}$, respectively.

acceleration voltage of the SEM in the range of $0.5-11 \mathrm{kV}$ and have not observed any significant change of the transport behavior in this range.

As mentioned previously, it has been demonstrated that electron irradiation creates sulfur vacancies in $\mathrm{MoS}_{2}{ }^{12}$ While the electron beam energy used in our experiment $(5 \mathrm{keV})$ may be too low to create defects by electron-beam sputtering process, ${ }^{33}$ beam damage through inelastic scattering process still occurs even at low energy through, e.g., a radiolysis effect ${ }^{33}$ and can create defects in $\mathrm{MoS}_{2}$ under SEM. ${ }^{6}$ Moreover, as reported by Egerton et al., ${ }^{33}$ radiolysis is temperature dependent and can be reduced by cooling the sample, and this is consistent with fact that our sample is less subjected to the electronic irradiation damage at low temperature $T=140 \mathrm{~K}$ although a charging effect is still noticeable at low temperatures.

It is noted that a transition from a bandlike electronic transport to transport via thermally activated mechanism, a metal to insulator transition, has been observed in $\mathrm{MoS}_{2}$ by tuning the carrier density or suppressing the interface charge effect. For example, Radisavljevic and Kis have demonstrated the use of the high- $k$ dielectric $\mathrm{HfO}_{2}$ as a top gate significantly improved the screening of the Coulomb interactions induced by the backgate $\mathrm{MoS}_{2} / \mathrm{SiO}_{2}$ interface. ${ }^{29}$ Consequently, a metallic transport with much improved mobility has been achieved. ${ }^{29}$ Similarly, in our case, high density of charge trap states can be created by the electron irradiation at the $\mathrm{MoS}_{2} / \mathrm{SiO}_{2}$ interface. The charge trap states may strongly screen the electric field of the backgate, leading to a constant insulating regime over the wide range of gate voltage and preventing a transition to the metallic regime. This observation highlights the important effect of electron beam, commonly used in imaging and fabrication techniques, on the device performance.

\section{SUMMARY}

We have studied temperature-dependent electrical transport in individual domains of monolayer $\mathrm{MoS}_{2}$ grown by CVD onto $\mathrm{Si} / \mathrm{SiO}_{2}$ substrates. We find that the electrons are mostly localized well up to room temperature and display multiple charge trapping and release behavior. We show electron irradiations can significantly affect the transport by increasing the carrier density and reducing the mobility. These effects are likely to arise from scattering by the intrinsic defects in $\mathrm{MoS}_{2}$ and the Coulomb potential of randomly distributed charges at the $\mathrm{MoS}_{2} / \mathrm{SiO}_{2}$ interface induced by electronic irradiation. Highly improved device performance should consider reducing defects in the active $\mathrm{MoS}_{2}$ layer, avoid electron beam irradiations, or suppressing the screening effect of the interface charges by appropriate device engineering.

\section{ACKNOWLEDGMENTS}

This research was conducted at the Center for Nanophase Materials Sciences, which is sponsored at Oak Ridge National Laboratory by the Scientific User Facilities Division, Office of Basic Energy Sciences, U.S. Department of Energy. A portion of theory work (C.D.) was supported by the Laboratory Directed Research and Development Program of Oak Ridge National Laboratory, managed by UT-Battelle, LLC, for the U. S. Department of Energy.

${ }^{1}$ A. Splendiani, L. Sun, Y. Zhang, T. Li, J. Kim, C.-Y. Chim, G. Galli, and F. Wang, Nano Lett. 10, 1271 (2010).

${ }^{2}$ S. Najmaei et al., Nat. Mater. 12, 754 (2013).

${ }^{3}$ H. Liu, M. Si, S. Najmaei, A. T. Neal, Y. Du, P. M. Ajayan, J. Lou, and P. D. Ye, Nano Lett. 13, 2640 (2013).

${ }^{4}$ A. M. van der Zande et al., Nat. Mater. 12, 554 (2013).

${ }^{5}$ B. Radisavljevic, A. Radenovic, J. Brivio, V. Giacometti, and A. Kis, Nat. Nanotechnol. 6, 147 (2011).

${ }^{6}$ W. Zhou et al., Nano Lett. 13, 2615 (2013).

${ }^{7}$ X. Zou, Y. Liu, and B. I. Yakobson, Nano Lett. 13, 253 (2013).

${ }^{8}$ S. Ghatak, A. N. Pal, and A. Ghosh, ACS Nano 5, 7707 (2011).

${ }^{9}$ C. Durand, M. Berthe, Y. Makoudi, J.-P. Nys, R. Leturcq, P. Caroff, and B. Grandidier, Nanotechnology 24, 275706 (2013).

${ }^{10}$ S. Mathew et al., Appl. Phys. Lett. 101, 102103 (2012).

${ }^{11}$ T.-Y. Kim, K. Cho, W. Park, J. Park, Y. Song, S. Hong, W.-K. Hong, and T. Lee, ACS Nano 8, 2774 (2014).

${ }^{12}$ H.-P. Komsa, J. Kotakoski, S. Kurasch, O. Lehtinen, U. Kaiser, and A. V. Krasheninnikov, Phys. Rev. Lett. 109, 035503 (2012).

${ }^{13}$ X. Liu et al., Nat. Commun. 4, 1776 (2013).

${ }^{14}$ H.-P. Komsa, S. Kurasch, O. Lehtinen, U. Kaiser, and A. V. Krasheninnikov, Phys. Rev. B 88, 035301 (2013).

${ }^{15}$ T.-H. Kim, Z. Wang, J. F. Wendelken, H. H. Weitering, W. Li, and A.-P. Li, Rev. Sci. Instrum. 78, 123701 (2007).

${ }^{16}$ A.-P. Li, K. W. Clark, X. G. Zhang, and A. P. Baddorf, Adv. Funct. Mater. 23, 2509 (2013).

${ }^{17}$ H. Qiu et al., Nat. Commun. 4, 2642 (2013). 
${ }^{18}$ J. D. Fuhr, A. Saúl, and J. O. Sofo, Phys. Rev. Lett. 92, 026802 (2004).

${ }^{19}$ S. Qin, T.-H. Kim, Z. Wang, and A.-P. Li, Rev. Sci. Instrum. 83, 063704 (2012).

${ }^{20}$ K. W. Clark, X. G. Zhang, I. V. Vlassiouk, G. He, R. M. Feenstra, and A.-P. Li, ACS Nano 7, 7956 (2013).

${ }^{21}$ A. Botman, J. J. L. Mulders, and C. W. Hagen, Nanotechnology 20, 372001 (2009).

${ }^{22}$ S. Das, H.-Y. Chen, A. V. Penumatcha, and J. Appenzeller, Nano Lett. 13, 100 (2013).

${ }^{23}$ N. A. Roberts, J. D. Fowlkes, G. A. Magel, and P. D. Rack, Nanoscale 5, 408 (2013).

${ }^{24}$ S. Tongay et al., Nano Lett. 13, 2831 (2013).

${ }^{25}$ R. Yang, X. Zheng, Z. Wang, C. J. Miller, and P. X.-L. Feng, J. Vac. Sci. Technol., B 32, 061203 (2014).
${ }^{26}$ S. M. Sze, Physics of Semiconductor Devices (Wiley, New York, 1981).

${ }^{27}$ G. Horowitz, M. E. Hajlaoui, and R. Hajlaoui, J. Appl. Phys. 87, 4456 (2000).

${ }^{28}$ A. R. Völkel, R. A. Street, and D. Knipp, Phys. Rev. B 66, 195336 (2002).

${ }^{29}$ B. Radisavljevic and A. Kis, Nat. Mater. 12, 815 (2013).

${ }^{30}$ W. Zhu, T. Low, Y.-H. Lee, H. Wang, D. B. Farmer, J. Kong, F. Xia, and P. Avouris, Nat. Commun. 5, 3087 (2014).

${ }^{31}$ D. Jariwala, V. K. Sangwan, D. J. Late, J. E. Johns, V. P. Dravid, T. J. Marks, L. J. Lauhon, and M. C. Hersam, Appl. Phys. Lett. 102, 173107 (2013).

${ }^{32}$ K. Kaasbjerg, K. S. Thygesen, and K. W. Jacobsen, Phys. Rev. B 85, 115317 (2012).

${ }^{33}$ R. F. Egerton, P. Li, and M. Malac, Micron 35, 399 (2004). 